\title{
Sulle condizioni di validità dei metodi di sommazione di Gronwall $\left({ }^{*}\right)$.
}

\author{
Temoria di Lurg Amerio (Milano)
}

Smuto. - Si stuliano le condizioni di validitì dei metodi di sommazione (f, g) di GronwaLL. con ipotesi assai meno restrittive per le funzioni $\mathrm{f}$ e g di quelle fatte dal GroNTHALr stesso. Si mettono in luce, successivamente, varie propriet⿳亠丷厂 generali relative ai metodi $(\mathrm{f}, \mathrm{g})$, sotto le nuove condizioni. e si applieano questi alla teoria del prolungamento analitico di una serie di potenze

\section{Introduzione.}

Una vasta classe di procedimenti di sommazione delle serie è stata definita da T. H. GronwaLL [1], con le seguenti considerazioni.

Una funzione $z=f(w)$, definita e continua per $|w| \leq 1$, sia analitica per $|w| \leq 1$, eccetto al più pẹr $x=1$, e dia la rappresentazione biunivoca e conforme di $|w|<1$ in un campo aperto $D$ interno a $|z|<1$, facendo corrispondere $z=0$ a $w=0, \approx=1$ a $w=1$; la funzione inversa sia a sua volta regolare anche lungo il contorno $l$ di $D$. eccetto al più per $z=1$, e si abbia precisamente

$$
1-w=(1-z)^{2} \psi(z)
$$

con $\lambda \geq 1, \psi(z)$ analitica in $D$ e su $l, \psi(1)>0, \psi(0)=1$.

Sia poi $g(n)$ una funzione analitica, rappresentata per $|w|<1$ dalla serite

$$
g(v)=\sum_{0}^{\infty} b_{n} b^{n} \quad b_{n} \neq 0
$$

e avente l' espressione

(3)

$$
g(w)=(1-w)-\gamma+\gamma(x)
$$

c in $\alpha>0, \gamma(w)$ analitica per $|w| \leq 1$; si abbia inoltre $g(w) \neq 0$ per $|w|<1$.

Chiameremo queste le condizioni di Gromvall per le funzioni $f(x)$ e $g(x)$.

Ciò posto, una serie $\sum_{0}^{\infty} u_{\nu}$ è detta dal Growwate sommabile $(f, g)$ quando di Pisa.

(1) Lavoro eseguito presso il Seminario Matematico della Scuola Formale Superioro 
le quantità $U_{0}, U_{1}, \ldots U_{n}, \ldots$ che si ricavano dall'identità

$$
\stackrel{\infty}{\Sigma}_{0}^{\infty} u_{y} z^{\prime}=\frac{1}{g(p)} \sum_{0}^{\infty} U_{n} b_{n} w^{n}
$$

tendono, per $n \rightarrow \infty$, a un limite finito $s$ che si assume come somma generalizzata della serie data.

Come si vede, le operazioni con cui si passa dalle $u_{\nu}$ alle $U_{n}$ sono due. In una prima, con la trasformazione $z=f(w)$, si muta la serie $\sum_{0}^{\infty} u_{y} z^{\nu}$ in una

$$
\sum_{0}^{\infty} \gamma_{m} w^{m} \text {. }
$$

Successivamente, dalla (4) si ricava

$$
\sum_{0}^{\infty} \Upsilon_{n} w^{m}=\frac{1}{g(w)} \sum_{0}^{\infty} b_{n} U_{n} w^{n}
$$

da cui, per la (2),

$$
U_{n}=\sum_{0}^{n} \gamma_{m} \frac{b_{n-m}}{b_{n}}
$$

cioè si applica alla serie $\stackrel{\infty}{\Sigma}_{0}^{\infty} \gamma_{m}$ un procedimento di sommazione di NokRLUND.

Si noti, a proposito della (7), che siccome è $f(0)=0$, lo sviluppo di $z^{\prime}$ secondo le potenze di $w$ comincia con la $y$-ma potenza: si ha perciò

$$
U_{n}=\sum_{0}^{n} a_{n, \nu} u_{\nu}
$$

dove le $a_{n, y}$ dipendono dai coefficienti di $f(w)$ e $g(w)$, ma non dalle $u_{y}$.

I metodi di GronwaLL sono molto generali e comprendono vari procedimenti di sommazione presentatisi nell'analisi.

Ad esempio, il metodo $\left(x,(1-x)^{-\alpha}\right)$ è il metodo $(C, \alpha-1)$ di Cesaro, con $\alpha>0$; il metodo $\left(\frac{1-(1-w)^{\frac{1}{2}}}{1+(1-w)^{\frac{1}{2}}},(1-w)^{-\frac{1}{2}}\right)$ è stato studiato dal DE LA VALlée Poussin e successivamente generalizzato dal Gronwall.

Un altro metodo di Growwall si è presentato in un mio recente lavoro [2] ed è stato studiato e ampliato dal BIRINDELLi; in esso si ha

$$
U_{n}=\sum_{0}^{n} \frac{\left(\begin{array}{c}
n \\
\nu
\end{array}\right) v !}{n^{\nu}}-u_{\nu}
$$

ciò che equivale a porre, come si dimostra, $w=z e^{1-z}, g(w)=\frac{1}{1-z}$. 
Il Gronwall ha provato pei metodi $(f, g)$ vari teoremi importanti. Notiamo fra questi il primo in cui si dimostra che se il $\lim _{n \rightarrow \infty} U_{n}$ esiste finito, allo stesso valore tende la funzione

$$
\varphi(z)=\sum_{0}^{\infty} u_{\nu} z^{\nu}
$$

quando $z \rightarrow 1$, restando in un campo angoloso col vertice in $z=1$, di aper* tara $<\frac{\pi}{\lambda}$ e avente per bisettrice il segmento $1^{1-i} 0$.

In un secondo teorema si prova che, se è $\lambda>1$ e il contorno $l$ di $D$ ha in comune con la circonferenza $|z|=1$ il solo punto $z=1$, è sommabile $(f, g)$ ogni serie sommabile $(C, \delta)$ con $\delta>-1$.

Successivamente il BIRINDELLI [3] ha ripresi e accuratamente studiati i metodi di Gronwall, applicandoli fra l'altro alla sommazione della serie geometrica $\sum_{0}^{\infty}, \xi^{y}$ verso la somma $\frac{1}{1-\xi}$ e determinando il relativo campo di sommabilità. Di qui ha ricavato, sfruttando la formula integrale di CAUCHY, un campo aperto in cui è certamente sommabile una serie di potenze $\underset{0}{\aleph_{\nu}} a_{\nu} \xi_{\nu}$ ottenendone così il prolungamento analitico.

$$
* * *
$$

In questa Memoria si sono ampliate le condizioni di Gronwall relative alle funzioni $f(w)$ e $g(w)$; si è potuto così provare che le ipotesi (1) e (3) possono essere grandemente allargate, soprattutto se ei si limita al problema del prolungamento analitico di una serie di potenze.

Inoltire si è dimostrato, sotto condizioni larghissime per la $f(w)$ e la $g(w)$, un teorema analogo al primo teorema di Gronwall.

Infine si è completato il risultato del BtRINDEIAL, perchè si è individuato per una serie di potenze un campo aperto $\Omega$, internamente al quale è possibile la sommabilità, mentre non lo è esternamente (almeno nel caso in cui la funzione rappresentata in un intorno dell'origine dalla serie stessa sia uniforme oppure quando il campo $D$ risulti interno ai suoi omotetici rispetto all' origine, $\tau D$, con $\tau>1$, ciò che avviene, ad esempio, nei metodi preceden. temente ricordati).

In quanto segue ammetteremo sempre che la funzione $z=f(w)$ sia ana. litica per $|w|<1$, continua per $|w| \leq 1$ e stabilisca una corrispondenza biunivoca e conforme tra $|w|<1$ e un campo aperto $D$ semplicemente connesso in modo che sia $f(0)=0, f(1)=1$. 
Essendo poi $\frac{d z}{d w} \neq 0$ per $|w|<1$, la funzione inversa $w=w(z)$ sarà ana. litica in tutti i punti di $D$.

Indicheremo oon $\bar{D}$ il campo chiuso costituito dai punti di $D$ e del contorno $l$, di equazione $z=f\left(e^{i v}\right)$, che supporremo sia una linea di Jordan, la quale, per noti teoremi [4], risulterà percorsa in un determinato verso dal punto $z=f\left(e^{i v}\right)$, al variare di \& tra $-\pi$ e $\pi$; inoltre la corrispondenza tra i due campí chiusi $|w| \leq 1$ e $\bar{D}$ sarà biunivoca e continua. Diremo infine che una proprietà vale in $D$, o in $\bar{D}$. quando è verificata in tutti i punti di $D$, o di $\bar{D}$.

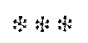

1. Condizioni relative alla funzione $a=f(w)$. - Cerchiamo in un primo tempo di ampliare le condizioni relative alla $z=f(x)$, considerando metodi di sommazione del tipo $\left(f,(1-x)^{-4}\right)$ con $\alpha>0$.

Per questo, posto

$$
(1-w)^{-x}={\underset{0}{\Sigma}}_{n}^{\infty} A_{n}^{(x)} w^{n}
$$

e inoltre

$$
\sum_{0}^{\infty} u_{y} z^{\nu}=\frac{1}{\left(1-k^{2}\right)-x} \sum_{0}^{\infty} V_{n z} A_{n}^{(x)} \eta^{n}
$$

dimostriamo il seguente

Teorema I. - Se il contorno 1 di $\mathrm{D}$ è ma curva rettificabile e ç(n) è ana. litica nel campo $\overline{\mathrm{D}}$, si ha

qurulunque sia $\alpha>0$.

$$
\lim _{n \rightarrow \infty} V_{n}=\varphi(1)
$$

Drmostraztone. - Posto $z=x-i y, \varphi(z)=\varphi_{1}(x, y)+i \varphi \cdot(x, y)$ dimostriamo che le due funzioni $\varphi_{1}(x, y), \varphi_{2}(x, y)$ sono a variazione limitata sul contorno $l$.

Infatti essendo $\varphi(\approx)$ analitica in tutti i punti del campo chiuso $\bar{D}$. per ogni $\bar{z}$ di $\bar{D}$ si può costruire un cerchio di raggio $\bar{\rho}>0$, finito, in modo che per $|z-\bar{z}| \leq \bar{\rho}$ sia $\varphi(z)$ analitica. Si ottiene così una famiglia di cerchi $e$ ogni punto di $\bar{D}$ risulta interno almeno a uno di essi : essendo l'insieme $D$ chíuso e limitato è possibile allora, per il lemma di Pincherde-Boret. co* struire con un numero finito di questi cerchi un campo ohiuso $\bar{B}$ tale che ogni punto di $\bar{D}$ sia interno a $\bar{B}$.

Sia $\lambda$ il contorno di $\bar{B}$ e $\delta>0$ la minima distanza dei punti di $l$ da $q$ uelli di $\lambda$. Dividiamo la curva $l$, di lunghezza finita $\nu$, in $n$ parti, rispettivamente di lunghezze $v_{1}, v_{2}, \ldots v_{n}$, in modo che sia $v_{i}<\delta$, mediante i punti $P_{0}=1, P_{1}, P_{2}, \ldots, P_{n-1}, P_{n}=1=P_{0}$; sarà evidentemente $\nu_{1}+\nu_{2}+\ldots+\nu_{n}=\nu$ 
Considerando dapprima la funzione $p_{1}(x, y)=\varphi_{1}(P)$ poniamo

$$
\omega_{n}={ }_{1}^{n}\left|\varphi_{1}\left(P_{n}\right)-\varphi_{1}\left(P_{r-1}\right)\right| \cdot
$$

Ora il segmento $P_{2-{ }_{1}} P_{r}^{-}$, essendo di lunghezza $d_{r} \leq \nu_{r}<\delta$, risulta interno a $B$; inoltre in $\bar{B}$ la funzione $\varphi_{1}(x, y)$ è armonica e quindi esiste una costante positiva $M$ tale che sia

$$
\left|\frac{\partial \varphi_{1}}{\partial x}\right| \leq M \quad\left|\frac{\partial \varphi_{1}}{\partial y}\right| \leq M
$$

Siccome poi è

$$
\left|\varphi_{1}\left(P_{r}\right)-\varphi_{1}\left(P_{r-1}\right)\right|=d_{r} \cdot\left|\frac{\partial \varphi_{1}\left(T_{r}\right)}{\partial s_{r}}\right|
$$

dove $T_{r}$ ̀̀ un punto interno del segmento $\overline{P_{r_{-1}} P_{r}}$ e $s_{r}$ ne è la direzione, si ricava per la $(13)$

$$
\left|\varphi_{1}\left(P_{r}\right)-\varphi_{1}\left(P_{r-1}\right)\right| \leq 2 M d_{r} \leq 2 M v_{r}
$$

perchè si ha

$$
\left.\left|\frac{\partial \varphi_{1}}{\partial s_{2}}\right|=\left|\frac{\partial \varphi_{1}}{\partial x} \cos (s, x)+\frac{\partial \varphi_{1}}{\partial y} \cos \right| s, y\right) \mid \leq 2 M
$$

Per la (15) si ricava dalla (12)

$$
\omega_{n} \leq 2 M L_{V}
$$

relazione che vale qualunque sia $n$ : infatti se per un numero $n_{u}$ di divisioni qualcuno dei segmenti $\overline{P_{r_{-1}} P_{n}}$ risulta di lunghezza $d_{r} \geq \delta$, è sempre possibile, coll'aggiunta di un numero finito di punti, sostituire al segmento stesso una spezzata $\mathrm{i}$ cui lati abbiano ciascuno lunghezza $<\delta$.

Compiuta la stessa operazione per tutti i segmenti $\overline{P_{r-1} P_{r}}$ di lunghezza $\geq^{\delta}$ e indicando con $n_{1}$ il numero di punti che così si ottengono, si ha evidentemente

$$
\omega_{n_{0}} \leq \omega_{n_{1}} \leq 2 M Y .
$$

Per la variazione $\omega$ di $\varphi_{1}(x, y)$ su $l$ si ha percio

$$
\omega \leq 2 M v
$$

cioè $\hat{\vartheta}_{1}(x, y)$ è a variazione limitata sul contorno stesso.

Allo stesso modo si ragiona per $\varphi_{2}(x, y)$.

Col cambiamento di variabile $z=f(x)$ la $\varphi(f(w))$ risulta analitica per $|w|<1$ : inoltre la sua parte reale e quella immaginaria risulteranno a variazione limitata sulla circonferenza $w=e^{i \vartheta}$, in virtù di quanto si $\dot{e}$ ora di- 
mostrato e del fatto che essendo $l$ una linea di JoRDAN, ai punti $P_{i}$ corrisponderanno sulla circonferenza $w=e^{i \vartheta}$ dei punti $Q_{i}$ che si seguixanno in on determinato verso al crescere di $\$$.

Posto allora

$$
\varphi(f(w))=\stackrel{\Sigma}{0}_{0}^{\infty} \gamma_{n} w^{n}
$$

il teorema sarà provato quando avremo dimostrato che la serie $\sum_{0}^{\infty} \gamma_{n}$ è som. mabile $(C, \alpha-1)$ con $x>0$.

Ora, posto $\gamma_{n}=\alpha_{n}-i \beta_{n}, w=\rho e^{i \vartheta}$, si ha per $p<1$

$$
\begin{aligned}
& \varphi(f(n))=\sum_{0}^{\infty} p_{n}^{n}\left\{\alpha_{n} \cos (n,)+\beta_{n} \operatorname{sen}(n y)\right\}+ \\
& +i \underset{0}{\infty} \Sigma_{n} p^{n}\left\{-\beta_{n} \cos (n \vartheta)+\alpha_{n} \operatorname{sen}(n \vartheta)\right\} \\
& =\psi_{1}(p, \vartheta)+i \psi_{2}(p, \psi)
\end{aligned}
$$

dove è

$$
\begin{aligned}
& \psi_{1}(\rho, \vartheta)=\varphi_{1}(x(\rho, \vartheta), y(\rho, \vartheta)) \\
& \psi_{2}(\rho, \vartheta)=\varphi_{2}(x(\rho, \vartheta), y(\rho, \vartheta)) .
\end{aligned}
$$

Dalla (18) si ricava in particolare

$$
\psi_{1}(\rho, 9)=\alpha_{0}+\sum_{1}^{\infty} \rho^{n}\left\{\alpha_{n} \cos (n g)+\beta_{n} \operatorname{sen}\left(n y^{\prime}\right)\right\}
$$

$\Theta$ quindi

$$
\begin{aligned}
& \alpha_{0}=\frac{1}{2 \pi} \int_{-\pi}^{\pi} \psi_{1}(\rho, \vartheta) d \vartheta \\
& \alpha_{n}=\frac{1}{\pi \rho^{n}} \int_{-\pi}^{\pi} \psi_{1}(\rho, \vartheta) \cos (n \vartheta) d \vartheta \\
& \beta_{n}=\frac{1}{\pi \rho^{n}} \int_{-\pi}^{\pi} \psi_{1}(\rho, \vartheta) \operatorname{sen}(n \vartheta) d \vartheta .
\end{aligned}
$$

Ma, essendo $\psi_{1}(\rho, 4)$ continua per $\rho \leq 1$, le (19) valgono anche se in esse si pone $\rho=1$; ne segue che le costanti $\alpha_{n}$ e $\beta_{n}$ sono i coefficienti di Fourier della funzione $\bigcup_{1}(1, \vartheta)$ la quale è, per quanto si è visto, continua e a varialimitata per $-\pi \leq \% \leq \pi$.

Per un noto teorema [5], la serie

$$
\alpha_{0}+\sum_{1}^{\infty}\left\{\alpha_{n} \cos (n 9)+\beta_{n} \operatorname{sen}(n g)\right\}
$$

¿े allora sommabile $(0, \alpha-1)$ con $\alpha>0$ e vale $\psi_{1}(1, y)$. 
Allo stesso modo si prova la sommabilità di

$$
-\beta_{0}+\sum_{1}^{\infty}\left\{-\beta_{n} \cos (n y)+\alpha_{n} \operatorname{sen}(n 9)\right\}
$$

che vale $\psi_{2}(1$. 计.

Posto poi nelle (20) e (21) $\vartheta=0$ ne segue che la serie $\sum_{0}^{\infty} \gamma_{n}$ è sommabile $(C, \alpha-1)$, con $\alpha>0$ e vale $\psi(1,0)+i \psi_{z}(1,0)=\varphi(1)$.

Il teorema è perciò dimostrato.

Facendo ora su $\alpha$ delle ipotesi più particolari possiamo provare altri due teoremi e precisamente:

Thorena II. - Se è $\alpha=1, \varphi(z)$ analitica nel campo $\overline{\mathrm{D}}$ e la linea $\mathrm{l}$ è rettificabile in un intorno di $\mathrm{z}=1$, si ha

$$
\lim _{n \rightarrow \infty} V_{n}=\varphi(1)
$$

Dnosmantone. - Pex le ipotesi fatte le funzioni $\psi_{1}(\rho, \vartheta), \psi_{2}(\rho, \vartheta)$ risul. tano continue per $\rho \leq 1$ e inoltre le $\psi_{1}(1,9), \psi_{2}(1$, q) sono a variazione limi. tata in un intorno di $\vartheta=0$.

Ne segue che le (19) valgono anche per $\rho=1$ e le serie (20) e (21) sono convergenti per $\mathfrak{q}=0$; da questo si deduce la tesi.

Teorema III. - Se è $\alpha>1, \varphi(\mathrm{z})$ analitica nel campo D, continua nel campo $\overline{\mathrm{D}}$, si ha

$$
\lim _{n \rightarrow \infty} V_{n}=\varphi(1)(*)
$$

Dimostraztone. - Bisognerà provare che la serie $\underset{\theta}{\Sigma_{n} \gamma_{n}}$ è sommabile $(C, \alpha-1) \operatorname{con} \alpha>1$.

Per questo osserviamo che, essendo $\approx=f(w)$ analitica per $|w|<1$, continua per $|w| \leq 1$, lo sarà, per le ipotesi fatte, anche la $\varphi(f(w))$; sono perciò funzioni continue per $\rho \leq 1$ tanto $\bigcup_{1}(\rho$, , $)$ quanto $\psi_{2}(\rho, 8)$.

Ne segue che le (19) continuano a valere se si pone in esse $p=1$ e quindi le $\alpha_{n}$ e $\beta_{n}$ sono $\mathrm{i}$ coefficienti di FourIer dello-sviluppo di $\psi_{1}(1,2)$. Essendo questa funzione continua, per un noto teorema [6] la serie (20) ̀̀ sommabile $(C, \alpha-1)$ con $\alpha>1$ e vale $\Psi_{1}(1, \vartheta)$.

Allo stesso modo si ragiona per la $\psi_{2}(1, \lambda)$.

Ponendo poi $\vartheta=0$ si ricava la sommabilità di $\sum_{0}^{\infty} \gamma_{n i}$ che avrà per valore $\psi_{1}(1,0)+i \psi_{2}(1,0)=\varphi(1)$.

$\left(^{*}\right) \varphi(1)$ il $\lim _{z \rightarrow 1} \varphi(z)$, quando $z \rightarrow 1$ restando in $\bar{D}$. 
Osservazione. - Nel caso in cui sia soddisfatta la condizione di Gron. WALL relativa alla $z=f(w)$ si puo facilmente dimostrare che valgono i teo. remi I, II, III.

Intatti la $z=f(w) \grave{e}$, in tale ipotesi, analitica per $|w| \leq 1, w \neq 1, \quad$ della (1) segue che in un intorno di $w=1$ vale uno sviluppo del tipo

$$
1-z=\widetilde{\Sigma}_{1} h_{n}(1-w)^{n}
$$

dove per $(1-w)^{\frac{1}{\lambda}}$ si prenderà quel ramo che si riduce a 1 per $w=0$.

Per la (22) la $z=f(w)$ è continua nel punto $w=1$; p(z) è perciò analitica in $D$, continua in $\bar{D}$, ed è applicabile il teorema $1 I I$.

Dimostriamo ora che il contorno $l$ di $D$ è rettificabile.

Infatti dalla (22) si ricava

$$
\frac{d z}{d w}=\sum_{1}^{\infty} k_{n} \frac{n}{\lambda}(1-w)^{\frac{n}{\lambda}}-1
$$

e, siccome per $w=e^{i v}$ si ha $|1-w|=2\left|\operatorname{sen} \frac{y}{2}\right|$, se ne deduce che $\left|\frac{d z}{d w}\right|$ diventa infinita come $\vartheta^{\frac{1}{2}-1}$ in prossimita di i $^{2}=0$.

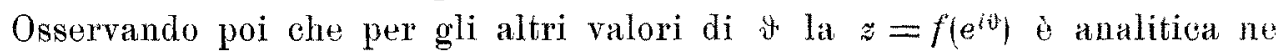
segue l'esistenza dell' integrale

$$
v=\int_{-\pi}^{\pi}\left|\frac{d z}{d w}\right||d w|=\int_{-\pi}^{\pi}\left|\frac{d z}{d x}\right| d y
$$

che rappresenta la lunghezza della linea $l$.

Vale percio il teorema I, e quindi il teorema II.

2. Condizioni relative alla fanzione $g(w)$. - Cerchiano ora di ampliaro lo condizioni relative alla $g(w)$.

Per questo, tenendo presenti le (10) e (11), poniamo

$$
\begin{aligned}
\sum_{0}^{\infty} u_{y} z^{\nu} & =\frac{1}{(1-w)^{-\infty}} \sum_{0}^{\infty} V_{n} A_{n}(x) w^{n} \\
& =\frac{1}{g(n)} \sum_{0}^{\infty} U_{n} b_{n} v^{n}
\end{aligned}
$$

cioè sommiamo la serie ${\underset{0}{\Sigma},}_{v}^{\infty}, z_{v}$ col metodo $\left(f,(1-w)^{-\alpha}\right)$ e col metodo $(f, g)$.

Ammesso che sia $\lim _{n \rightarrow \infty} V_{n}=s$, finito, cerchiamo le condizioni per cui è anche $\lim _{n \rightarrow \infty} U_{n}=s$. 
Posto allora

$(25)$

$$
g(x)=\sum_{0}^{\infty} b_{n} w^{n}=\frac{{\stackrel{\stackrel{\infty}{\Sigma}}{n_{n}} \Delta_{n}^{(\alpha)} w^{n}}_{0}^{0}}{(1-w)^{\alpha}} .
$$

si rienva

$$
\begin{aligned}
\Delta_{n}^{(x)} & =\sum_{0}^{n} b_{m} A_{m-m}^{(-x)} \\
b_{n} & =\sum_{0}^{n} \Delta_{m}^{(x)} A_{n-m}^{(\alpha)}
\end{aligned}
$$

dove $A_{n}^{(-\alpha)}$ è il coefficiente di $w^{n}$ nello sviluppo di $(1-w)^{x}$.

Ciò premesso, e ricordando che ̀̀ $A_{n}^{(x)}>0$, possiamo dimostrare il se. guente

Teorema IV. - Se la successione

$$
b_{0}, \quad b_{1}, \ldots, b_{n} \ldots
$$

soddisfa per $\alpha \geq 1$, alle condizioni

$$
\begin{aligned}
& \text { (a) } \quad \frac{\left|\sum_{n}^{n} \Delta_{m}^{(x)} A_{n-m}^{(x)}\right|}{\left|\sum_{n}^{n}\right| \Delta_{m}^{(x)}\left|A_{n-m}^{(x)}\right|}=\frac{\left|b_{n}\right|}{\left|\sum_{m}^{n}\right| \Delta_{m}^{(x)}\left|A_{n-m}^{(\alpha)}\right|}>M>0 \\
& \text { b) } \quad \lim _{n \rightarrow \infty} \frac{b_{n-1}}{b_{n}}=1
\end{aligned}
$$

ed ̀̀ $\lim \mathrm{V}_{n}=\mathrm{s}$, finito, si ha pure

$$
\lim _{n \rightarrow \infty} U_{n}=s .
$$

Dimostrazione. - Si ricava dalle (24) e (25)

$$
J_{n}=\sum_{0}^{n} V_{m} \frac{A_{m}^{(\alpha)} \Delta_{n-m}^{(\alpha)}}{b_{n}}
$$

e le condizioni necessarie e sufficienti perohè, essendo $\lim _{n \rightarrow \infty} V_{n}=s$, sia anche $\lim _{n \rightarrow \infty} U_{\hat{n}}=s$, come è noto [7], sono:

1) $\lim _{n \rightarrow \infty} \frac{\Delta_{n-m}^{(x)}}{b_{n}}=0$ per $m=0,1, \ldots$

2) $\sum_{0}^{n} A_{m}^{(x)}\left|\frac{\Delta_{n-m}^{(x)}}{b_{n}}\right|<K>0$ per $n=0,1, \ldots$

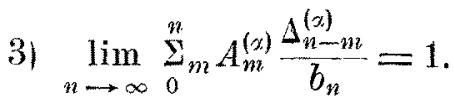

La condizione 3) è identicamente soddisfatta per la (27). 
Quanto alla 2) si constata immediatamente che coincide con la condi. zione a) e che è $M=\frac{1}{K}$.

Resta da provare che, con lo ipotesi a) e b) è soddisfatta la 1).

Per questo osserviamo che si ha per le (27) e $a$ )

$$
\left|\frac{b_{m}}{b_{n}}\right|<\frac{\sum_{r}^{m}\left|\Delta_{r}^{(x)}\right| A_{m-r}^{(x)}}{M \sum_{0}^{n}\left|\Delta_{r}^{(x)}\right| A_{n-r}^{(x)}}=K \frac{A_{m}^{(x)}}{\bar{A}_{n}^{(x)}} \frac{\sum_{r}^{m}\left|\Delta_{r}^{(x)}\right| \frac{A_{m-r}^{(\alpha)}}{\sum_{m}^{(x)}}}{\sum_{r}^{n} \mid \Delta_{r}^{(x)} \cdot \frac{A_{n-r}^{(x)}}{A_{n}^{(x)}}}
$$

E poi, per $m \geq 1$,

$$
\begin{aligned}
A_{m}^{(\alpha)} & =(-1)^{m}\left(\begin{array}{r}
-\alpha \\
m
\end{array}\right)=\frac{\alpha(\alpha+1) \ldots(\alpha+m-1)}{1 \cdot 2 \ldots m} \\
& =\left(1+\frac{\alpha-1}{1}\right)\left(1+\frac{\alpha-1}{2}\right) \ldots\left(1+\frac{\alpha-1}{m}\right)
\end{aligned}
$$

e quindi

$$
\frac{A_{m}^{(\alpha)}}{A_{m-r}^{(r)}}=\left(1+\frac{\alpha-1}{m-r+1}\right) \cdots\left(1+\frac{\alpha-1}{m}\right) .
$$

Ne segue, se $\dot{\theta} \alpha \geq 1$, per $m \leq n$,

$$
\frac{A_{m-r}^{(\alpha)}}{A_{m}^{(\alpha)}} \leq \frac{A_{m-r}^{(\alpha)}}{A_{n l}^{(\bar{x})}}
$$

e, per la (29),

$$
\left|\frac{b_{m}}{b_{n}}\right|<K \frac{A_{m}^{(\alpha)}}{A_{n}^{(x)}}
$$

Osserviamo ora che dalla condizione b) segue, per un dato $m$,

$$
\lim _{n \rightarrow \infty} \frac{b_{n-m}}{b_{n}}=\lim _{n \rightarrow \infty} \frac{b_{n-m}}{b_{n-m+1}} \ldots \frac{b_{n-1}}{b_{n}}=1
$$

perciò, siccome ̀े

$$
\frac{\Delta_{n}^{(x)}}{b_{n}-m}=\frac{b_{n-m}-\frac{\Delta_{n}}{(\alpha)}}{b_{n}} \frac{m}{b_{n-m}}
$$

posto $n-m=N$, per provare la 1), basterà dimostrare che si ha

$$
\lim _{N \rightarrow \infty} \frac{\Delta_{N}^{(\alpha)}}{b_{N}}=\lim _{N \rightarrow \infty} \sum_{0}^{N} \frac{b_{N}-m}{b_{N}} A_{n z}^{(-\alpha)}=0
$$

Ora è, per $m \geq 1$,

$$
A_{m}^{(-\alpha)}=(-1)^{m}\left(\begin{array}{c}
\alpha \\
m
\end{array}\right)=\frac{[(m-1)-\alpha][(m-2)-\alpha] \ldots[-\alpha]}{m !} .
$$


E poi $r-1<\alpha \leq r$, con $r$ intero positivo; ne segue che per $0 \leq m \leq r$ i termini della successione $A_{0}^{(-x)}=1, A_{1}^{(-x)}, \ldots, A_{m}^{(-x)}, \ldots$ hanno segni alternati, mentre per $m>r, A_{m}^{(-x)}$ ha lo stesso segno di $A_{r^{(-\alpha)}}^{(o p p u r e ~ e ̀ ~ n u l l o ~ s e ~ e ̀ ~}$ $x=r$ ).

Inoltre si ha (*)

$$
\stackrel{\infty}{0}_{m} A_{m}^{(-x)}=0
$$

A preso $\varepsilon>0$ si può determinare $p>r$ in modo che per $N \geq p$ sia sempre

$$
\left|\sum_{m}^{N} A_{m}^{(-x)}\right|=\sum_{p}^{N}\left|A_{m}^{(-x)}\right|<\frac{\varepsilon}{2(K+1)}
$$

e quindi, pex la (37)

$$
\left|\sum_{0}^{p-1} A_{m}^{\left(-x_{1}\right)}\right| \leq \frac{\varepsilon}{2(K+1)} .
$$

Ora si ha, per $N \geq p$,

$$
\left|\sum_{0}^{N} \frac{b_{N-m}}{b_{N}} A_{m}^{(-x)}\right| \leq\left|\sum_{0}^{p-1} \frac{b_{N-m}}{b_{N}} A_{m}^{(-x)}\right|+\stackrel{-}{\Sigma}_{p}^{N}\left|\frac{b_{N-n}}{b_{N}}\right|\left|A_{m}^{(-x)}\right|
$$

e quindi, per la (33),

$$
\sum_{0}^{N}\left|\frac{b_{N-m}}{b_{N}} A_{m}^{(-\alpha)}\right|<\left|\sum_{0}^{p-1} \frac{b_{N-m}}{\Sigma_{N}} A_{m}^{(-\alpha)}\right|+K||_{p}^{N} \frac{A_{N-m}^{(\alpha)}}{A_{N}^{(\alpha)}} A_{m}^{(-\alpha)} \mid .
$$

Siccome poi è

$$
(1-w)^{x} \frac{1}{(1-w)^{x}}=\sum_{0}^{\infty} w^{N} \sum_{0}^{N} A_{N-m}^{(x)} A_{m}^{(-x)} \equiv 1,
$$

si ricava, per $N \geq 1$,

$$
{\stackrel{N}{\Sigma_{m}}}_{0}^{N} A_{N-m}^{(\alpha)} A_{m}^{(-\alpha)}=0
$$

(*) Osserviamo che, per $r-1<x<r, m>r$, si ricava dalla formula di Stinlinca

$$
\begin{aligned}
A_{m}^{(-\alpha)} & =\frac{(-x)(-\alpha+1) \ldots(-x+r-1)}{\Gamma^{\prime}(r-\alpha)} \frac{\Gamma(m-\alpha)}{\Gamma(m+1)} \\
& =\frac{(-x)(-\alpha+1) \ldots(-x+v-1)}{\Gamma^{\prime}(r-x)} \frac{(m-\alpha-1)^{m-\alpha-1+\frac{1}{2}} e^{-(m-x-1)+\frac{\vartheta^{\prime}}{12(m-\alpha-1)}}}{m^{m+\frac{1}{2}} e^{-m+\frac{\vartheta^{\prime \prime}}{12 m}}}
\end{aligned}
$$

con $0<\vartheta^{\prime}<1,0<\vartheta^{\prime \prime}<1$.

Ne segue

$$
\lim _{m \rightarrow \infty} \frac{A_{m}^{(-\alpha)}}{m^{-(x+1)}}=\frac{(-x)(-x+1) \ldots(-x+r-1)}{\Gamma(r-x)} .
$$


e quindi, per la $(40)$,

$$
\begin{gathered}
\left|\sum_{0}^{N} \frac{b_{N-m}}{b_{N}} A_{m}^{(-x)}\right|<\left|\sum_{0}^{p-1} \frac{b_{N-m}}{b_{N}} A_{m}^{(-x)}\right|+ \\
+K\left|\sum_{0}^{p-1} \frac{A_{N-n}^{(x)}}{\sum_{N}^{(\alpha)}} A_{m}^{(-x)}\right| \rightarrow(1+K)\left|\sum_{0}^{p-1} A_{m}^{(-x)}\right|
\end{gathered}
$$

in virtù della condizione $b$ ) e della (32).

Si puó allora determinare $N_{0} \geq p$ in modo che per $N>N_{0}$ sia, per la (39),

$$
\left|\sum_{m}^{N} \frac{b_{N-m}}{b_{N}} A_{m}^{(-\alpha)}\right|<\varepsilon
$$

e quindi il teorema è completamente provato.

Nel caso in eni sia $0<\alpha<1$ vale il seguente

Teorema V. - Se la successione

$$
b_{0}, \quad b_{1}, \ldots, \quad b_{,}, \ldots
$$

soddisfa, per $0<\alpha<1$. alle condizioni a) e b) del teorema I I' e inoltre alla condizione

$$
\text { c) }\left|\frac{b_{m}}{b_{n}}\right|<K_{1} \frac{A_{m}^{(\varkappa)}}{A_{m}^{(\alpha)}},
$$

$m \leq n$

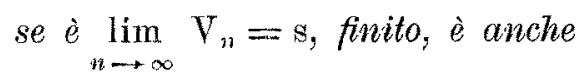

$$
\lim _{n \rightarrow \infty} U_{n}=s
$$

Dimostrazione. - Basterà provare che, mediante le condizioni poste si ha

$$
\lim _{N \rightarrow \infty} \frac{\Delta_{N}^{(x)}}{b_{N}}=0
$$

ciò che è immediata conseguenza della condizione c) e delle (40), (41), (42), (43), (44), nelle quali si ponga $K$, in luogo di $K$.

Osservazione. - Dimostriamo che se la $g(w)$ soddisfin alla condizione (3) di Gronwall, soddisfa anche alle condizioni $a$ ) e $b$, se è $\alpha \geq 1 ; a$, b), c) se è $0<\alpha<1$.

Infatti in un tal caso is $b_{n}=A_{n}^{(x)}+-\beta_{n}$ con $\beta_{n} \mid<B \tau^{n}, B>0$ e $0<\tau<1$. per l'analiticita di $\gamma(w)$ per $|w| \leq 1$.

Ne segue immediatamente che è soddisfatta la condizione b).

Quando alla c) osserviamo che ò

$$
\frac{A_{n}^{(\alpha)}}{A_{m}^{(\alpha)}}\left|\frac{b_{m}}{b_{n}}\right|=\frac{1+\frac{\beta_{m}}{A_{m}^{(x)}}}{1+\frac{\beta_{n}}{A_{n}^{(\alpha)}}}
$$$$
m \leq n
$$ 
a $\lim _{m \rightarrow \infty} \frac{\beta_{t i}}{A_{m}^{(x)}}=0$. Percio il numeratore $s$ superiormente limitato; quanto al denominatore, ricordando che $\dot{\theta} b_{n} \neq 0$, si può affermare che rimane supe. viore a un numero $\partial>0$; la condizione c) è perciò verificata.

Considerando la condizione a) si ha, per la (25)

$$
\begin{aligned}
& \stackrel{ }{n}_{n}^{\infty} \Delta_{z t}^{(x)} w^{n}=1+(1-w)^{x} \gamma(w) \\
& =1+\stackrel{\Sigma}{\Sigma}_{n}^{\infty} w^{n}{\underset{v}{0}}_{m}^{n} \beta_{n-m n} A_{m}^{(-\infty)}
\end{aligned}
$$

e, per la convergenza assoluta degli sviluppi di $(1-w)^{\alpha}$ e di $\gamma(w)$ per $w=1$ ne segue la convergenza di $\sum_{0}^{\infty}\left|\Delta_{n}^{(x)}\right|$; per un noto teorema la serie stessa sarà perciò sommabile $(C, x-1)$ con $\alpha \geq 1$.

Dimostriamo che $\dot{\theta}$ anche sommabile $(C, \alpha-1)$ con $\alpha>0$.

Per questo basterà [8] dimostrare che ò $n\left|\Delta_{i l}^{(\alpha)}\right|=0$ (1), cioè, per la (47),

$$
n\left|\sum_{0}^{n} \beta_{n-m} A_{m}^{(-0)}\right|<k
$$

dove $k$ è una costante positiva.

Ora si ha, indicando con $q$ il più piccolo intero $\geq \frac{n}{2}$,

$$
\begin{aligned}
& n\left|\sum_{0}^{n} \beta_{n-m} A_{m}^{(-\alpha)}\right| \leq n B \underset{0}{\sum_{n}^{q} \tau^{n-n}}\left|A_{m}^{(-\alpha)}\right|+n B \underset{q+1}{\sum_{m}} \tau^{n-m}\left|A_{m}^{(-\alpha)}\right| \\
& \leq n B \tau^{n-q}{\underset{\theta}{\Sigma}}_{m}^{\infty}\left|A_{m}^{(-x)}\right|+n B_{1} n^{-(x+1)} \sum_{0}^{\infty} \tau^{m} \rightarrow 0
\end{aligned}
$$

dove $B_{1}$ è un numero positivo (*).

La (48) è perciò dimostrata.

Ritornando alla condizione a) osserviamo che deve essere

cioè

$$
\frac{b_{n} \mid}{\sum_{n}\left|\Delta_{m}^{(x)}\right| A_{m-m}^{(x)}}>M>0
$$

$$
\frac{\left|1+\frac{\beta_{n}}{A_{n}^{(x)}}\right|}{\sum_{0}^{n}\left|\Delta_{m}^{(x)}\right| \frac{A_{n-m}^{(x)}}{A_{n}^{(x)}}}>M
$$

(*) V. teorema IV, (*). 
eiò che avviene certamente perchè il numeratore $\rightarrow 1$ (ed è $\left.b_{n} \neq 0\right)$ e il denominatore resta limitato essendo la serie $\sum_{0}^{\infty} \mid \Delta_{n z}^{(x)}$ sommabile $(C, \alpha-1)$ con $\alpha>0$.

3. Proprietà generali dei metodi $(f, g)$. - a) Tenendo conto dei teoremi IV e $\mathrm{V}$ si possono generalizzare i teoremi I, II, III.

Si ha cosi:

Thorema VI. - Se il contorno 1 di D è una curva rettificabile e la $\mathrm{g}(\mathrm{w})$ soddisfa alle condizioni del teorema $V$, la serie ${\underset{0}{D}}_{\nu}^{\infty} \mathrm{u}_{,}$è sommabile $(\mathrm{f}, \mathrm{g})$ e vale supposta la $\varphi(\mathrm{z})$ analitica nel campo $\overline{\mathrm{D}}$.

Teorema VII. - Se il contorno 1 è rettificabile in un intomo di $\mathrm{z}=1 e$ la $\mathrm{g}(\mathrm{w})$ soddisfa alle condizioni

$$
\begin{array}{cc}
\lim _{n \rightarrow \infty} \frac{b_{n-1}}{b_{n}}=1 & b_{n} \neq 0 \\
\left|b_{0}\right|+\sum_{n}^{\infty}\left|b_{m}-b_{m-1}\right|<K\left|b_{n}\right| &
\end{array}
$$

la serie $\sum_{0}^{\infty} \mathrm{u}_{v}$ è sommabile (f, g) e vale $\varphi(1)$, supposta la $\varphi(\mathrm{z})$ analitica nei punti di $\overline{\mathrm{D}}$.

Teorema VIII. - Se la g(w) soddisfa alle condizioni del teorema IV per un valore $\alpha>1$, la serie $\sum_{0}^{\infty} \mathrm{u}_{v}$ è sommabile (f, g) e vale

$$
\varphi(1)=\lim _{z \rightarrow 1} \varphi(z)
$$

quando la $\varphi(\mathrm{z})$ sia analitica in $\mathrm{D}$, continua in $\overline{\mathrm{D}}$, e $\mathrm{z} \rightarrow 1$ restando in $\overline{\mathrm{D}}$.

b) Una notevole conseguenza del teorema VIII è il seguente

Tworema IX. - Se il contorno 1 di D ha tutti $i$ punti, eccetto $\mathrm{z}=1$, in. terni alla circonferenza $|\mathrm{z}|=1$, e inoltre il campo $\mathrm{D}$ è interno a un campo angoloso $\mathrm{T}$ col vertice in $\mathrm{z}=1$, di ampiezza $<\pi$ e avente per bisettrice il segmento $1^{1-i} 0$, è sommabile (f, g) ogni serie $\sum_{0}^{\infty} \mathrm{u}_{v}$ la quale sia sommabile (C, $\delta)$ (con un valore $\delta>-1$ ), quando la $\mathrm{g}(\mathrm{w})$ soddisfi alle condizioni del teo. rema IV per un valore $\alpha>1$.

Drmostrazione. - Supposto che la serie $\sum_{0}^{\infty}, u_{\nu}$ sia sommabile $(C, \delta)$, con $\delta>-1$, e converga al valore $s$, sarà, per un noto teorema, $\lim _{z \rightarrow 1} f(z)=s$, quando $z \rightarrow 1$ in $T$ e quindi, a maggior ragione, restando nel campo $\bar{D}$. Essendo poi tutti i punti di $\bar{D}$, escluso $z=1$, interni alla circonferenza $|z|=1$, si può affermare che la $\varphi(z)$ è continua in $\bar{D}$, analitica in $D$. 
Applicando il teorema precedente si ricava allora

$$
\lim _{n \rightarrow \infty} U_{n}=s .
$$

Osservazione. - A proposito del teorema IX bisogna notare che due metodi $\left(f_{1}, g_{1}\right)$ e $\left(f_{2}, g_{2}\right)$, soddisfacenti entrambi alle condizioni in esso richieste con due valori $\alpha_{1}>1, \alpha_{2}>1$, possono attribuire valori diversi a una serie $\sum_{0}^{\infty} u_{\nu}$ la quale non sia sommabile $(C, \delta)$ per nessun $\delta>-1$.

Si ponga ad esempio $u_{\nu}=(-1)^{\nu} \frac{1}{\sqrt{2}}\left(\begin{array}{l}1 \\ 2 \\ v\end{array}\right) 2^{\nu}$, sicchè sarà $\varphi(z)=\sqrt{\frac{1}{2}-z}, \Theta$ supponiamo che il campo $\overline{D_{1}}$ relative alla $f_{i}(z)$, pur soddisfacendo alle condizioni del teorema IX, non contenga il punto singolare $z=\frac{1}{2}$ ma sia.tale che per $R(z)=\frac{1}{2}$ si abbia pei suoi punti $I(z)>0$.

Ora quando $z \rightarrow 1$ in $\bar{D}_{1}$ la funzione $\sqrt{\frac{1}{2}-z}$, analitica nei punti di $\bar{D}_{1}$, $\rightarrow \frac{-i}{\sqrt{2}} ;$ si ha perciò

$$
\lim _{n \rightarrow \infty} U_{n}^{(1)}=-\frac{i}{\sqrt{2}}
$$

Se adesso supponiamo che il campo $\overline{D_{2}}$, relativo a $f_{2}(z)$, sia costituito dai punti coniugati di quelli di $\bar{D}_{1}$, quando $z \rightarrow 1$, restando in $\bar{D}_{2}$, la funzione $\sqrt{\frac{1}{2}-z}$, analitica nei punti di $\bar{D}_{2}, \rightarrow \frac{i}{\sqrt{2}}$ e quindi si ricava

$$
\lim _{n \rightarrow \infty} U_{n}^{(2)}=\frac{i}{\sqrt{2}}
$$

c) Nei teoremi VI, VII, VIII si pone in relazione l'analiticità di $\varphi(z)$ in $D$ o in $\bar{D}$ colla sommabilità $(f, g)$ della serie $\sum_{0}^{\infty} u_{y}$, relazione che è ulterior* mente precisata dal

TEorema X. - Se $\lim _{n \rightarrow \infty} \mathrm{U}_{n}$ esiste finito e la funzione $\mathrm{g}(\mathrm{w})$ è analitica e $\neq 0$ per $|\mathrm{w}|<1$, la $\varphi(\mathrm{z}) \grave{e}$ analitica in tutti i punti del campo D.

Drmostrazione. - Siccome esiste finito il $\lim _{n \rightarrow \infty} U_{n}$, esiste una costante positiva $M$ per cui è

e quindi

$$
\left|U_{n}\right| \leq M
$$

$$
\left|U_{n} b_{n}, w^{n}\right| \leq M\left|b_{n} w^{n}\right|
$$


Siccome poi per $|w|<1$ converge la serie

$$
\sum_{0}^{\infty}\left|b_{n} w^{n}\right|
$$

per la (49) sarà pure convergente la

$$
\sum_{0}^{\infty} U_{i} b_{i ;} w^{n}
$$

e rappresenterà una funzione analitica per $\mid w<1$.

Ne segue, ricordando che è $g(w) \neq 0$ per $|w|<1$, l'analiticità nello stesso campo di

$$
\frac{1}{g(w)}{ }_{0}^{\infty} U_{n} U_{n} b_{n} w^{n}
$$

cioè, per la (6), di

$$
\varphi(f(w))={\underset{\Sigma}{\Sigma}}_{m}^{\infty} \gamma_{m} w^{m}
$$

e quindi di $\varphi(z)$ in $D$, a causa della corrispondenza binnivoca e conforme tra il cerchio $|w|<1$ e il campo $D$.

d) Sia $\sigma$ una linea continua, aperta, avente un estremo in $z=1$. Diremo che la serie $\underset{0}{\Sigma_{y}} u_{\nu}$ è sommabile con un metodo di PoIsson gentralizzato $(P, \sigma)$, quando esiste finito il

$$
\lim _{z \rightarrow 1} \varphi(z)
$$

supposto che $z \rightarrow 1$ lungo o; come valore della serie si assumerà il limite stesso.

Ciò premesso, un'estensione del $1^{0}$ teorema di GronwaLl è data dal seguente

Teorema XI. - Se la $\mathrm{g}(\mathrm{w})$ soddisfa alle condizioni del teorema $X$ e per $i$ coefficienti $\mathrm{b}_{n}=\beta_{n}+i \bar{o}_{n}$ del suo sviluppo si ha $\beta_{n}>0$ per $\mathrm{n}>\mathrm{n}_{0}$ sufficien. temente grande, $\sum_{0}^{\infty} \beta_{n}=+\infty, \sum_{0}^{\infty} \mid \delta_{n \mid}=\mathrm{k}$, finito; e se la serie $\sum_{0}^{\infty} \mathbf{u}_{v}$ è sommabile (f, g), essa ̀̀ anche sommabile (P, o), con lo stesso valore, quando come linea $\sigma$ si prenda quella di equazione $\mathrm{z}=\mathrm{f}(\mathrm{w})$, con $0 \leq \mathrm{w} \leq 1$.

Dimostrazione. - Posto $s=\lim _{n \rightarrow \infty} U_{n}$, pel teorema $X$ la funzione $\varphi(z)$ è analitica in $D$. Ool cambiamento di variabile $z=f(w)$, essa risulta analitica per $|w|<1$ e si ha

$$
\varphi(f(w))=\frac{\sum_{n}^{\infty} U_{n} b_{n} w^{n}}{g(w)}
$$


da eui

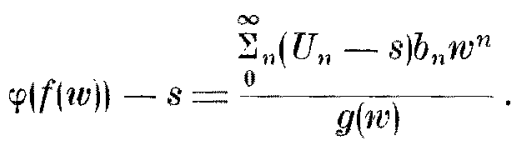

Fissato $\varepsilon>0$, per $n>n_{1}$ sarà $\left|U_{n}-s\right|<\frac{\varepsilon}{3}$; preso $N$ maggiore del più grande tra i numeri $n_{0}$ e $n_{i}$, siccome è $\lim _{n \rightarrow 1} \sum_{N}^{\infty} \beta_{n} w^{n}=+\infty$ quando $w \rightarrow 1$ assu. mendo valori reali, per $0 \leq w_{0} \leq w<1$ sarà $\sum_{N}^{\infty} \beta_{n} w^{n}>k+\sum_{0}^{N-1} \mid b_{n}$, e si avrà

$$
|\varphi(f(w))-s| \leq \frac{{\stackrel{N}{\Sigma_{n}} \mid}_{0}^{N-1}\left|\left(U_{n}-s\right) b_{n}\right|+\frac{\varepsilon}{3} k+\frac{\varepsilon}{3}{\underset{N}{\Sigma}}_{n}^{\infty} \beta_{n} w^{n}}{\sum_{N}^{\infty} \beta_{n} w^{n}-\left(k+\sum_{0}^{N-1}\left|b_{n}\right|\right)} .
$$

Posto $A={\underset{0}{\Sigma}}_{0}^{N-1}\left|\left(U_{n}-s\right) b_{n}\right|+\frac{\varepsilon}{3} k, B=k+\frac{N-1}{\Sigma_{n}}\left|b_{n}\right|$, sarà allora

$$
|\varphi(f(w))-s| \leq \frac{A}{\sum_{N}^{\infty} \beta_{n} w^{n}-B}+\frac{\varepsilon}{3} \frac{1}{1-\frac{B}{\sum_{N}^{\infty} \frac{B}{\sum_{n} \beta_{n} w^{n}}}}<\frac{\varepsilon}{2}+\frac{\varepsilon}{2}=\varepsilon
$$

quando sia $w_{0} \leq w_{1} \leq w<1$.

Ricordando che la funzione $z=f(w)$ è continua per $|w| \leq 1$, e quindi per $w_{1} \leq w \leq 1$, ne segue la tesi.

Da questo teorema si ricava che, nelle ipotesi fatte, il metodo di somma. zione $(f, g)$ non è più potente del metodo di Porsson generalizzato.

Inoltre se una serie è sommabile coi metodi $\left(f, g_{1}\right)$ e $\left(f, g_{2}\right)$ i valori che si ottengono sono eguali, mentre, come risulta dall' osservazione che segue al teorema IX, non sono eguali in generale $i$ valori ottenuti con due metodi $\left(f_{1}, g_{1}\right),\left(f_{2}, g_{2}\right)$.

Bisogna però ora osservare che nella grande maggioranza dei metodi di sommazione usati in pratica (ad esempio in quelli ricordati nell' introduzione) i coefficienti dello sviluppo di $z=f(w)$ (e così pure i $b_{n}$ ), sono reali e quindi la linea $\sigma$ è costituita dal segmento $0^{1-1} 1$.

Ne segue che, se si fa una tale ipotesi su $f_{1}(w)$ e $f_{2}(w)$, supposta la serie

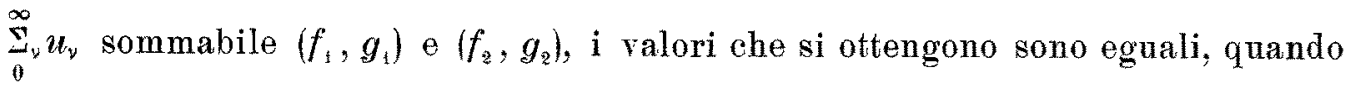
naturalmente $g_{1}(w)$ e $g_{2}(w)$ soddisfino alle condizioni del teorema XI.

Ora queste, per quel che riguarda i $b_{n}$, sono certo verificate se i $b_{n}$ 
sono reali e si ha

$$
\lim _{n \rightarrow \infty} \frac{b_{n-1}}{b_{n}}=1 \quad\left|b_{n}\right|>H A_{n}^{(\alpha)} \quad \alpha>0
$$

dove $H$ è una costante positiva, ciò̀ quando abbiano luogo le $b$ ) e (33) del teorema IV o le b) e c) del teorema $V$.

Infatti in tal caso per $n>n_{0}$ sufficientemente grande i $b_{n}$ avranno tutti lo stesso segno, per esempio positivo (se fosse $b_{n}<0$, basterebbe cambiar segno alle $g(w)$, il che non altera il valore di $\left.U_{n}\right)$; sarà allora per $n>n_{0}$,

$$
b_{n}>H A_{n}^{(\alpha)}
$$

e la serie $\sum_{n}^{\infty} b_{n}$ divergerà $\mathbf{a}+\infty$.

\section{I metodi $(f, g)$ e il prolungamento analitico di nna serie di potenze.} - Nel trattare l'applicazione dei metodi $(f, g)$ al prolungamento analitico di una serie di potenze faremo l'ipotesi che le funzioni $f(w)$ e $g(w)$ soddisfino rispettivamente alle condizioni del teorema I (contorno $l$ rettificabile) e dei teoremi V e X. Si supporrà inoltre che tutti i punti della linea $l$ escluso $z=1$ siano interni alla circonferenza $|z|=1$.

Si abbia ora la serie di potenze

$$
F(z)=\sum_{0}^{\infty} a_{\nu} z^{\psi}
$$

e si dia $a z$ un valore $\xi \neq 0$. Indicando con $\xi D$ e $\xi \bar{D}$ i campi che si ottengono da $D$ e $C$ moltiplicandone i punti per $\xi$, possiamo dimostrare il

TEorema XII. - Se la funzione $\mathrm{F}(\mathrm{z})$ è analitica in $\overline{\mathrm{\zeta}} \mathrm{D}$ la serie $\sum_{0}^{\infty} \mathrm{a}_{\nu} \xi^{2}$ è sommabile (f, g) e vale $\mathrm{F}(\xi)$.

Se in $\xi \mathrm{D}$ esiste qualche singolarità della $\mathrm{F}(\mathrm{z})$ la serie stessa non è sommabile (f, g).

Drmostrazione. Se $F(z)$ è analitica in $\xi \bar{D}$, posto $u_{y}=\alpha_{\nu} \xi^{\nu}$, la funzione $\varphi(z)=\sum_{0}^{\infty} u_{y} \hat{z}^{\prime}$ è analitica in $\bar{D}$ e pel teorema VI la serie $\sum_{0}^{\infty} u_{\nu}$ è sommabile $(f, g)$ e vale $\varphi(1)=F(\xi)$.

Se in $\xi D$ esiste qualche singolarità della $F(z)$, in $D$ esisterà qualche singolarità della $\varphi(z)$ e, pel teorema $X$, la serie $\stackrel{\sim}{*}_{0}^{\infty} u$, non sarà sommabile $(f, g)$.

Supponiamo ora che la $F(z)$ abbia una sola singolarita al finito nel punto $z=\xi_{0}$. 
Indicata con $l^{-1}$ la linea di equazione

$$
z=\frac{1}{f\left(e^{\mathrm{i} \vartheta}\right)}
$$$$
-\pi \leq \vartheta \leq \pi
$$

dimostriamo che la serie $\sum_{0}^{\infty}, a_{\nu} \xi^{\nu}$ è sommabile $(f, g)$, e vale $F(\xi)$, per $\xi$ appartenente al campo aperto $A_{0}$, avente per contorno la linea $\xi_{0} l^{-1}$, mentre non ̀े sommabile $(f, g)$ se $\xi$ è esterno ad $A_{0}$.

Infatti, pel teorema $\mathrm{X}$, la sommabilità si ha nei punti $\xi$ per cui è $\xi f(w) \neq \xi_{0}$ per ogni valore $w$ con $|w| \leq 1$, cioè $\xi \neq \frac{\xi_{0}}{f(w)}$ e quindi appartenente ad $A_{0}$; la sommabilità viene a mancare nei punti $\xi$ per cui esista un $w_{1}$, con $\left|w_{\xi}\right|<1$, tale che si abbia $\xi f\left(w_{1}\right)=\xi_{0}$, cioè per $\xi$ esterno ad $A_{0}$.

Nel caso in cui la funzioné $F(z)$ abbia più di un punto singolare, ma sia uniforme, si indichi con $\xi_{i}$ un generico punto singolare e con $A_{i}$ il campo aperto costituito dai punti interni alla linea $\xi_{i} l^{-1}$ e con $Q$ l'insieme costituito dai punti comuni a tutti gli $A_{i}$.

Si dimostra [9] che $\Omega$ è un eampo aperto semplicemente connesso.

Se $\xi \grave{e}$ in $\Omega$, nessun punto singolare $\xi_{i}$ della $F(z)$ cadrà nel campo $\xi \bar{D} \mathrm{e}$ la serie $\sum_{0}^{\infty} a_{\nu} \xi^{\nu}$ sarà sommabile $(f, g)$ e varrà $F(\xi)$; se $\xi$ è esterno a $Q$ almeno un punto singolare $\xi_{i}$ della $F(z)$ cadrà in $\xi D$ e verrà a mancare la sommabilità $(f, g)$ della serie stessa.

Inoltre, per l'ipotesi fatta sulla linea $l$, ad $\Omega$ appartengono i punti di analiticità della $F(z)$ non esterni alla circonferenza di convergenza della serie $\sum_{0}^{\infty} a_{\nu} z^{\nu}$

Nel caso in cui la $F(z)$ (considerata come complesso di tutti i suoi ele. menti) non sia uniforme potranno esistere dei suoi punti singolari $\bar{\xi}_{p}$ che cadano internamente alla circonferenza di convergenza, di raggio $R$, della serie $\sum_{0}^{\infty} a_{\nu} z^{y}$.

A questi punti si giunge però eseguendo il prolungamento analitico lungo linee $\bar{\sigma}_{p}$ che partano da $z=0$ e avvolgano dei punti singolari non interni alla circonferenza stessa.

Sia $\xi_{i}$ un punto singolare della $F(z)$, soddisfacente alla condizione $\left|\xi_{i}\right| \geq R$ e $\Omega^{\prime}$ il campo aperto, semplicemente connesso, costituito dai punti comuni a tutti gli $A_{i}$ relativi ai punti $\xi_{i}$.

Il campo $\Omega^{\prime}$ contiene manifestamente i punti $\bar{\xi}_{p}$, però siccome non si può tracciare nessuna linea $\bar{\sigma}_{p}$ che non sia parzialmente esterna a $\Omega^{\prime}$, gli $\bar{\xi}_{p}$ non 
sono da considerare come punti singolari $e$ in $\Omega^{\prime}$ la $F(z)$ risulta analitica $e$ uniforme.

Se allora $\xi$ appartiene a $\alpha^{\prime}$, siccome nel campo $\xi \bar{D}$ non può cadere nessun punto $\xi_{i}$, ne segue la sommabilità $(f, g)$ di $\sum_{0}^{\infty} a_{\nu} \xi^{y}$ che varrà $F(\xi)$.

Un altro campo $\Omega^{\prime \prime}$ in cui è sicuramente possibile la sommabilità $(f, g)$ della serie data, è stato indicato dal BirIndelur [10].

Precisamente si ha che, se l'elemento di funzione analitica si può prolungare analiticamente in un eampo semplicemente connesso $\Sigma$, dando luogo a un ramo monodromo $F(\xi)$, la serie data sara sommabile $(f, g)$ verso la somma $F(\xi)$ per $\xi$ interno all'insieme $S A_{0}$, dove $S$ è l'insieme complementare di $\Sigma$, rispetto al piano $z$.

Nel caso in cui $\Sigma$ è la stella rettilinea, $S$ è costituito dai punti $\tau \xi_{i}$. dove $i$ punti $\xi_{i}$ sono certi punti singolari del ramo (quelli che si incontrano nel prolungamento analitico della $F(z)$ lungo semirette spiccate da $z=0$ ), ed è $\tau \geq 1$. La sommabilità sarà allora possibile per $\xi$ interno a tutti i campi $\xi_{i} A_{0}$ e ai loro omotetici rispetto all'origine con rapporto $\tau>1$.

E chiaro che il ragionamento del BIRINDELLI equivale al considerare come punti singolari tutti i punti di $S$.

Se $Q$ è il campo aperto, costituito dai punti appartenenti a uno almeno dei campi $\Omega^{\prime}$ e $\mathbf{\Omega}^{\prime \prime}$, possiamo affermare che, per $\xi$ in $\Omega$, la serie $\sum_{0}^{\infty} a_{\nu} \xi^{y}$ è sommabile $(f, g)$ e vale $F(\bar{g})$.

Supponiamo ora che il campo $D$ sia interno ai suoi omotetici rispetto all' origine, con rapporto $\tau>1$. In tal caso, assumendo come campo $\Sigma$ la stella rettilinea, $Q^{\prime \prime}$ sarà costituito dalla parte comune a tutti i campi $\xi_{i} A_{0}$; inoltre conterrà $\Omega^{\prime}$ e quindi coinciderà con $\Omega$.

Se allora $\xi$ appartiene a $\Omega$, la serie $\sum_{0}^{\infty} a_{\nu} \xi_{\nu}$ è sommabile $(f, g)$ e vale $F(\xi)$; se invece $\xi$ è esterno a $\Omega$, cade entro $\xi D$ almeno uno dei punti $\xi_{\text {, e }}$ il raggio vettore che lo congiunge a $z=0$ : ne segue, pel teorema XII, che la serie data non è sommabile $(f, g)$.

\section{BIBLIOGRAFIA}

1. T. H. GronwatL, Summation of series and conformal mapping. "Annals of Mathematics"* Second series, Vol. 33, 1932.

2. L. AMERIO. Un metodo di sommazione per le serie di potenze e sua applicazione alla tenria della trasformazione di Laplace, "Ann. Se. N. di Pisa", Serie II, 1939.

3. C. Brrinderis, Contributo all'analisi dei metodi di sommazione di Gronwall, "Rend. Cire. Mat. di Palermo *, Tomo LXT, 1988, p. 157, 176. 
4. C. Caratheodorx, Conformal representation, "Cambridge Tracts s, 1932, p. 86.

5. E. Kogbetuixz, Summation des serves et intégrales divergentes par les moyennes ari. tmétiques et typiques, "Mem. des Sc. Math.». p. 66, Paris, Gauthier-Villars, 1931.

6. G. H. Hardy: On the summability of Fourier"s series, "London Math. Soc. Proc. ", Vol. 12, 1913, p. 365̃. 372.

7. O. Tomplitz, Ueber allgemeine lineare Mittelbildungen, “Prace mat.-fiz. ", Vol. 22, 1913, p. $113,119$.

8. G. H. HARdy e J. E. Lrtwhewood, Contributions to the anthmetic theory of series, "London Math. Soc. Proc.», Vol. 11, 1911, p. 462, 463.

9. C. Binindelur, Sull'applicazione dei metodi di sommazione di Gronwall al problema del prolungamento analitico, "Ann. Sc. N. di Pisa», Serie IT, Vol. VI, 1937, p. 182. 10. C. Birindelti, v 3, p. $170,171$. 